Arq. Bras. Med. Vet. Zootec., v.59, n.3, p.627-633, 2007

\title{
Obstrução recorrente das vias aéreas em muares: relato de três casos
}

\author{
[Airway recurrent obstruction in mules: report of three cases] \\ U.P. Melo ${ }^{1}$, C. Ferreira ${ }^{2}$, M.S. Palhares ${ }^{3 *}$ \\ ${ }^{1}$ Faculdade de Castelo - Castelo, ES \\ ${ }^{2}$ Aluna de pós-graduação - EV-UFMG - Belo Horizonte, MG \\ ${ }^{3}$ Escola de Veterinária - UFMG \\ Caixa Postal 567 \\ 30123-970 - Belo Horizonte, MG
}

\begin{abstract}
RESUMO
Relatam-se três casos de obstrução recorrente das vias aéreas em muares com idade média de 10 anos. Os animais eram utilizados para concurso de marcha e criados em campo. Após serem mantidos em cocheiras com cama de serragem e alimentados com feno (tifton e alfafa) e ração comercial, começaram a manifestar intolerância ao exercício e episódios de tosse durante o exercício. Após exames clínico e laboratorial, instituiu-se terapia à base de clenbuterol, dexametazona e bromexina, além de controle ambiental. Após 21 dias de tratamento, ocorreu remissão dos sintomas clínicos. Para comprovação diagnóstica, os animais foram submetidos ao desafio ambiental, por um período de dois dias. Após o tratamento, os três animais voltaram a desempenhar suas atividades atléticas de modo satisfatório.
\end{abstract}

Palavras-chave: muar, obstrução recorrente, vias aéreas, intolerância ao exercício

\begin{abstract}
Three cases of airway recurrent obstruction in approximately 10-year-old mules are reported. The animals were raised free and used for marching competitions. After being stabled in boxes under a wood scrape bed and fed on tifton and alfalfa hay plus a commercial ration, they started to show intolerance to exercise and episodes of coughing during it. After clinical and laboratorial examinations, clenbuterol, dexametazone and bromexine were administrated, besides controlling the environment. After 21 days of treatment, the clinical symptoms ceased. In order to certify that diagnosis, the animals were submitted to an environmental challenge for two days. Then, the animals were back to their normal athletic activities in a satisfactory manner.
\end{abstract}

Keywords: mules, recurrent obstruction, airways, exercise intolerance

\section{INTRODUÇ̃̃O}

A inflamação do complexo traqueobrônquico acompanhada de obstrução é uma ocorrência comum nos eqüinos, especialmente os mantidos em cocheiras por longos períodos com dieta à base de feno (Robinson et al., 1996; Léguillette, 2003). Na literatura, há relatos de sua ocorrência em asininos (Thiemann e Bell, 2001). Essa síndrome de inflamação e obstrução tem recebido diferentes nomenclaturas, incluindo

Recebido em 11 de julho de 2006

Aceito em 20 de abril de 2007

*Autor para correspondência (corresponding author)

E-mail: palhares@vet.ufmg.br bronquite crônica, bronquiolite crônica, doença das pequenas vias áreas, obstrução recorrente das vias áreas (RAO), porém é conhecida mundialmente como doença pulmonar obstrutiva crônica (DPOC) (Mair e Derksen, 2000).

O termo DPOC, utilizado na medicina humana, descreve a doença pulmonar inflamatória em fumantes, com patofisiologia e evolução clínica completamente diferente daquela vista nos eqüinos (Art et al., 1998; Léguillette, 2003). Após constantes esforços de pesquisadores, 
determinou-se a nomenclatura correta para as doenças inflamatórias das vias aéreas dos eqüinos. Atualmente pode-se fazer a distinção entre a síndrome dos eqüinos jovens (animais com até cinco anos de idade), de caráter reversível, chamada doença inflamatória das vias aéreas (IAD) e a obstrução crônica das vias áreas, de caráter irreversível, nos eqüinos adultos (animais com idade superior a oito anos) chamada de asma ou RAO (Léguillette, 2003).

A RAO tem sido definida como uma doença ocupacional dos eqüinos, resultante das condições sob as quais são mantidos para treinamento (Derksen, 1993). A enfermidade é observada em eqüinos no mundo inteiro e tende a acometer animais com idade média de oito anos (Ainsworth e Biller, 2000), não existindo predileção por raça ou sexo (Davis e Rush, 2002). O achado característico dessa enfermidade é a reversibilidade dos sinais clínicos quando os animais são mantidos em ambiente livre de poeira ou quando retornam à pastagem (Légguillette, 2003).

A manifestação inicial da doença pode ser subclínica, e os eqüinos podem se mostrar alertas e sem febre. O único sintoma pode ser tosse ocasional durante alimentação ou no inicio do exercício. Com a progressão para o quadro clínico típico, a freqüência e a intensidade dos episódios de tosse aumentam (Davis e Rush, 2002). A manifestação clínica clássica varia de intolerância ao exercício a dispnéia crônica durante repouso (Moore, 1996). Os eqüinos apresentam-se com tosse crônica, corrimento nasal mucoso a mucopurulento, narinas dilatadas e dispnéia expiratória acentuada (Rush, 1997).

Essas alterações podem ser sazonais e mais intensas quando os eqüinos são mantidos em cocheiras e expostos a feno de qualidade ruim e com muita poeira, esporos fúngicos, vapor de amônia e outros estímulos não específicos. O estabulamento de eqüinos afetados pode levar ao agravamento do quadro clínico em menos de três dias, manifestado principalmente pelo inicio abrupto de episódios freqüentes de tosse (Robinson et al., 2003).

Este trabalho tem por objetivo relatar a ocorrência e o tratamento da obstrução recorrente das vias aéreas em três muares marchadores.

\section{CASUÍSTICA}

Três muares marchadores, fêmeas, com idades estimadas de 10 anos (caso 1), 12 anos (caso 2) e oito anos (caso 3), sem histórico anterior de qualquer enfermidade sistêmica, foram examinados para diagnóstico da etiologia de tosse crônica e intolerância ao exercício.

Os animais eram utilizados para concursos de marcha e foram adquiridos de três propriedades distintas, em épocas diferentes, onde eram mantidos em campo. Após a compra, todos foram mantidos em cocheiras com cama de serragem e alimentados com feno de tifton e alfafa, além de ração concentrada ( $1 \mathrm{~kg}$ ração/100kg de peso vivo). Os três animais eram desvermifugados trimestralmente e imunizados contra raiva (casos 1, 2, e 3), influenza (casos 1, 2, e 3), tétano (casos 1 e 2), encefalomielite (casos 1 e 3 ) e rinopneumonite (casos 1, 2, e 3).

Quando estabulados, começaram a manifestar episódios de tosse durante o período de alimentação e, com o decorrer do tempo, durante o exercício (casos 1 e 2). Os principais sintomas relatados foram intolerância ao exercício (casos 1 e 2), tosse crônica durante a alimentação (casos 1,2 e 3) e exercício (caso 1), além de perda de peso (caso 3). Os sintomas clínicos apresentavam sete meses de duração no caso 1 , nove meses de duração no caso 2 e seis meses de duração no caso 3.

Após a anamnese, realizou-se exame clínico completo, conforme mostrado na Tab. 1. Nesse exame inicial, os animais foram submetidos a 45 minutos de exercício, o qual mimetizou as condições de prova a que os mesmos eram submetidos; decorrido esse tempo, realizou-se outro exame clínico. Os resultados do exame clínico pósexercício são mostrados na Tab. 2.

Após o exame clínico, foi realizado hemograma, em que todos os parâmetros apresentaram-se dentro da normalidade, exceto o aumento no valor da hemoglobina. Além do hemograma, realizou-se o exame de fezes (ovos por grama de fezes - OPG) para descartar a presença de vermes pulmonares (D. arnifield).

Baseando-se na anamnese e nos achados do exame clínico, tanto em repouso quanto em exercício, suspeitou-se de obstrução recorrente das vias aéreas. 
Obstrução recorrente das vias aéreas...

Tabela 1. Achados do exame clínico dos três muares em repouso

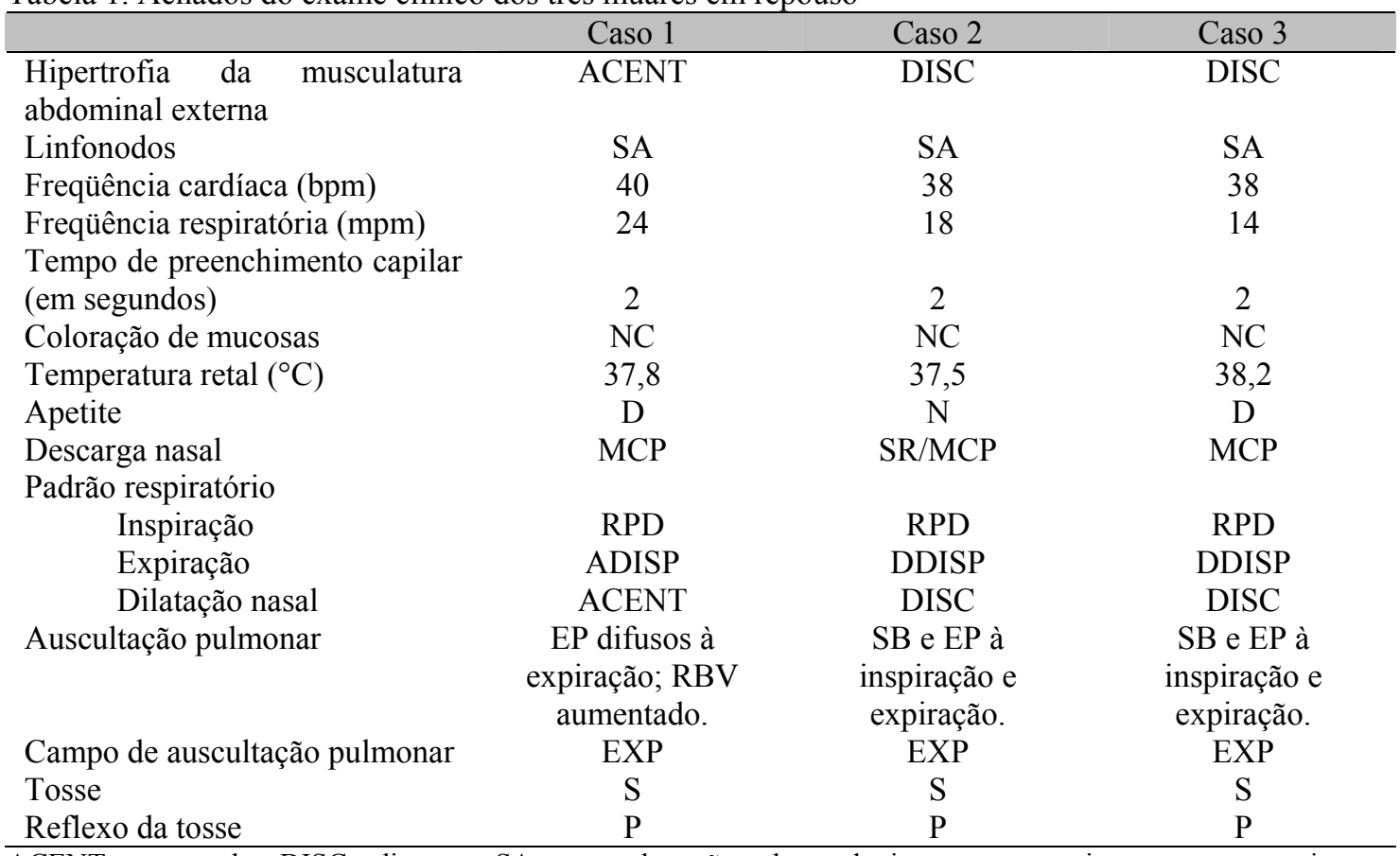

ACENT: acentuada; DISC: discreta; SA: sem alterações; bpm: batimentos por minuto; mrm: movimentos respiratórios por minuto; MCP: mucopurulenta; SR; serosa; NC: normocoradas; RPD: rápida; ADISP: acentuada dispnéia; DDISP: discreta dispnéia; D: diminuído; N: normal; SB: sibilos; EP: estertor pulmonar; RBV: ruído broncovesicular; EXP: expandido; S: seca; P: presente.

Tabela 2. Achados do exame clínico dos três muares pós-exercício

\begin{tabular}{|c|c|c|c|}
\hline & Caso 1 & Caso 2 & Caso 3 \\
\hline Freqüência cardíaca (bpm) & 185 & 150 & 140 \\
\hline Freqüência respiratória (mpm) & 70 & 50 & 55 \\
\hline $\begin{array}{l}\text { Tempo de preenchimento capilar } \\
\text { (em segundos) }\end{array}$ & 2 & 2 & 2 \\
\hline $\begin{array}{l}\text { Tempo de recuperação cardíaca } \\
\text { (em minutos) } \\
\text { Tempo de recuperação }\end{array}$ & 20 & 10 & 7 \\
\hline respiratória ${ }^{\mathrm{a}}$ (em minutos) & 30 & 15 & 8 \\
\hline Coloração de mucosas & $\mathrm{CG}$, discreto $\mathrm{HC}$ & $\mathrm{NC}$ & $\mathrm{NC}$ \\
\hline Temperatura retal $\left({ }^{\circ} \mathrm{C}\right)$ & 42 & 41 & 42 \\
\hline \multicolumn{4}{|l|}{ Padrão respiratório } \\
\hline Inspiração & RPD & RPD & RPD \\
\hline Expiração & ADISP & MDISP & DDISP \\
\hline Dilatação nasal & $\mathrm{P}$ & $\mathrm{P}$ & $\mathrm{P}$ \\
\hline Auscultação pulmonar & $\begin{array}{l}\text { EP difusos à } \\
\text { inspiração e } \\
\text { expiração }\end{array}$ & $\begin{array}{l}\text { SB e EP à } \\
\text { inspiração e } \\
\text { expiração }\end{array}$ & $\begin{array}{l}\text { SB e EP à } \\
\text { inspiração e } \\
\text { expiração }\end{array}$ \\
\hline Tosse & $\begin{array}{l}\text { Seca, quatro a oito } \\
\text { episódios/minuto } \\
\text { durante o exercício }\end{array}$ & $\begin{array}{l}\text { Seca, um a dois } \\
\text { episódios/minuto } \\
\text { durante o exercício }\end{array}$ & $\begin{array}{l}\text { Seca, cinco } \\
\text { episódios durante o } \\
\text { exercício }\end{array}$ \\
\hline
\end{tabular}


Após o diagnóstico clínico, os animais foram tratados com: (1) Clenbuterol $^{1}(0,8 \mu \mathrm{g} / \mathrm{kg})$, via oral a cada 12 horas durante dois dias, seguidos de $3,2 \mu \mathrm{g} / \mathrm{kg}$ administrados pela mesma via e com o mesmo intervalo de tempo durante 18 dias; (2) dexametazona $^{2}(0,1 \mathrm{mg} / \mathrm{kg} \mathrm{IV})$, a cada 24 horas durante três dias, seguidos de $0,05 \mathrm{mg} / \mathrm{kg}$ IV durante três dias e $0,02 \mathrm{mg} / \mathrm{kg}$ IV durante três dias; (3) cloridato de bromexina ${ }^{3}(0,3 \mathrm{mg} / \mathrm{kg} \mathrm{IV})$, a cada 24 horas durante oito dias; (4) controle ambiental, que consistiu em alocar os animais em baias com piso de borracha e umedecimento do feno por 20 minutos antes do fornecimento, durante cinco dias, e alocação dos mesmos em piquetes de tifton após esse período.

Após 21 dias de tratamento, os animais foram reavaliados, seguindo o mesmo protocolo do exame inicial (Tab. 3), e submetidos a 45 minutos de exercício. Nesse segundo teste, os animais não apresentaram intolerância ao exercício ou episódios de tosse, e os parâmetros clínicos encontraram-se dentro da normalidade.

Para comprovar o diagnóstico de RAO, os três animais foram submetidos ao desafio ambiental, consistindo de seu retorno às cocheiras com cama de serragem e alimento à base de feno sem umedecimento, por um período de dois dias. Após 12 horas de estabulamento, dois deles apresentaram episódios de tosse seca (casos 1 e 2), e um começou a tossir 26 horas após o desafio ambiental. Após 36 horas de desafio, os três apresentavam dispnéia expiratória e secreção nasal mucopurulenta (casos 1 e 2) e mucosa (caso 3). Na auscultação pulmonar, observaramse sibilos inspiratórios ou, ao final da fase expiratória, crepitações e estertores traqueais, sons bronco-vesiculares aumentados, além de prolongamento da fase expiratória da respiração.

Tabela 3. Achados do exame clínico dos três muares após 21 dias de tratamento

\begin{tabular}{lccc}
\hline & Caso 1 & Caso 2 & Caso 3 \\
\hline Freqüência cardíaca (bpm) & 36 & 34 & 38 \\
Freqüência respiratória (mpm) & 14 & 12 & 10 \\
Tempo de preenchimento capilar (em segundos) & 2 & 2 & 2 \\
Coloração de mucosas & $\mathrm{NC}$ & $\mathrm{NC}$ & $\mathrm{NC}$ \\
Temperatura retal $\left({ }^{\circ} \mathrm{C}\right)$ & 37,5 & 37,8 & 37,2 \\
Inapetência & $\mathrm{A}$ & $\mathrm{A}$ & $\mathrm{A}$ \\
Descarga nasal & $\mathrm{A}$ & $\mathrm{A}$ & $\mathrm{A}$ \\
Padrão respiratório & & & $\mathrm{N}$ \\
$\quad$ Inspiração & $\mathrm{N}$ & $\mathrm{N}$ & $\mathrm{N}$ \\
$\quad$ Expiração & $\mathrm{DDISP}$ & $\mathrm{N}$ & $\mathrm{SA}$ \\
Auscultação pulmonar & $\mathrm{EP} / \mathrm{LCE}$ & $\mathrm{SA}$ & $\mathrm{A}$ \\
Tosse & $\mathrm{A}$ & $\mathrm{A}$ & $\mathrm{A}$ \\
Reflexo da tosse & $\mathrm{A}$ & $\mathrm{A}$ & \\
\hline
\end{tabular}

Bpm: batimentos por minuto; mpm: movimentos por minuto; NC: normocorada; A: ausente; N: normal; DDISP: discreta dispnéia; EP: poucos estertores pulmonares no lobo caudal esquerdo; SA: sem alteração

\section{DISCUSSÃO}

$\mathrm{Na}$ maioria dos casos de RAO, o diagnóstico pode ser realizado com base no histórico e nos achados clínicos (Mair e Derksen, 2000; Davis e Rush, 2002; Légguillette, 2003). Nesses relatos, o diagnóstico foi baseado nos mesmos parâmetros, além dos exames laboratoriais e

\footnotetext{
${ }^{1}$ Pulmonil Gel, Vetnil, Louveira, São Paulo

${ }^{2}$ Dextar - União Química Farmacêutica Nacional, EmbuGuaçu, São Paulo

${ }^{3}$ Aliv V - União Química Farmacêutica Nacional, EmbuGuaçu, São Paulo
}

respostas à terapia e ao desafio ambiental. O resultado do OPG e ausência de $D$. arnifield indicou que o distúrbio pulmonar não era de origem parasitária.

A RAO é freqüentemente diagnosticada em eqüinos e pode acometer asininos. Não foram encontrados relatos na literatura consultada quanto à ocorrência em muares. A provável razão disso deve-se à menor freqüência dessa espécie em atividades eqüestres, além do fato de rotineiramente serem criados em regime de pasto. No Brasil, principalmente nos Estados de 
Minas Gerais, São Paulo e Paraná, esses animais são utilizados em concursos de marcha e exposições, quando permanecem a maior parte do dia em cocheiras recebendo dietas à base de feno (tifton ou alfafa), que os predispõem ao desenvolvimento da RAO.

Outras causas de tosse e/ou intolerância ao exercício foram descartadas durante a anamnese e o exame clínico, tendo como base o histórico dos animais, a natureza crônica da doença, as condições de manejo da propriedade e a regressão dos sinais clínicos após medidas de controle ambiental para redução do desafio alergênico.

Há polêmica em relação à causa da RAO. Muitos a consideram uma reação de hipersensibilidade à poeira ou a fungos comumente encontrados no feno e/ou no material da cama, sendo tal condição relativamente rara em animais mantidos em pasto. Os três fungos mais comumente implicados são Aspergillus fumigatus, Microspolyfora faeni, além do Termoactinomyces vulgaris (Ainsworth e Biller, 2000; Mair e Derksen, 2000).

Embora os três animais fossem mantidos em ambiente bem ventilado, o quadro clínico de obstrução recorrente das vias aéreas desenvolveu-se após a estabulação. Feno e material de cama têm muita poeira, propícios ao desenvolvimento de fungos termofílicos e esporos de actinomicetos. Muitos desses esporos são pequenos o bastante para alcançarem os bronquíolos e dentro deles depositarem quando aspirados pelos eqüídeos, iniciando uma reação inflamatória por efeitos irritantes primários (Mair e Derksen, 2000).

Como o feno é uma fonte de esporos lesivos, a concentração desses antígenos é maior onde se manipula o feno. O comportamento característico do eqüídeo, ao se alimentar, é o de sacudir o feno e comê-lo por longos períodos, o que resulta em concentração mais elevada de poeira na área respiratória - espaço aéreo ao redor da narina do que no restante da baia (Mair e Derksen, 2000), sugerindo ser esta a causa da enfermidade nos animais deste relato.

Nos eqüinos afetados pela RAO, com quadro clínico avançado, o diagnóstico é baseado principalmente nos sintomas. O clínico deve descartar uma pneumonia infecciosa primária ou secundária, que possa estar contribuindo para a disfunção pulmonar (Davis e Rush, 2002). Os hemogramas dos três animais não apresentaram indício de doença de natureza infecciosa, descartando pneumonia como uma possível causa. $\mathrm{O}$ aumento observado no valor da hemoglobina está de acordo com os achados de Art et al. (1998), estando associada à necessidade de o animal afetado equilibrar a troca gasosa pulmonar deficiente.

Eqüinos afetados desenvolvem quadro de disfunção da ventilação pulmonar e do fluxo sangüíneo. Isso provoca troca gasosa ineficiente, hipoxemia e hipercapnia, devido à obstrução difusa das vias aéreas inferiores. Para compensar a troca gasosa ineficiente, os animais afetados aumentam sua ventilação/minuto por meio do aumento da freqüência respiratória, conforme observado nos três casos descritos. Para inalar o mesmo volume tidal, no mesmo espaço de tempo, há necessidade de uma taxa de fluxo de ar médio mais elevada em face da obstrução das vias aéreas, justificando o surgimento do padrão respiratório típico demonstrado pelo animal afetado (Nyman et al., 1991; Tremblay et al., 1993; Art et al., 1998; Robinson et al., 2000; Holcombe et al., 2001).

O quadro de disfunção pulmonar e a troca gasosa ineficiente provocados pela doença explicam a intolerância ao exercício demonstrada nos três casos, bem como os parâmetros clínicos observados após o exercício. A observação de discreto halo-cianótico do caso 1 provavelmente originou-se da hipoxemia e hipercapnia, embora nos presentes casos não tenha sido realizada gasometria sangüínea para comprovação laboratorial.

Para diminuir a exposição à poeira e a fatores alergênicos, todas as baias receberam o mesmo manejo adotado para as baias dos animais afetados. A unificação do feno, em uma tentativa de diminuir a concentração de poeira na zona respiratória, apresentou resultados satisfatórios, embora a literatura recomende a sua retirada da dieta (Vandenput et al., 1998). A diminuição da exposição à poeira é essencial para a terapia a longo prazo. Os fármacos administrados possuem apenas efeitos transitórios se medidas especificas de controle da poeira da baia ou do ambiente não forem adotadas (Lavoie, 1997). 
A reversibilidade dos sinais clínicos após mudanças rígidas no manejo pode demorar de três a quatro semanas. Esse tempo de remissão está relacionado à idade do eqüino, a duração da obstrução das vias aéreas e à gravidade da doença (Lavoie, 1997). O uso prolongado de broncodilatores sem adoção de rígidas medidas de controle ambiental pode aumentar a quantidade de alérgenos que alcança as pequenas vias aéreas, agravando a inflamação (Davis e Rush, 2002; Léguillette, 2003).

A administração de corticosteróides melhora os sinais clínicos, reduz a inflamação das vias aéreas, beneficiando a função pulmonar nos eqüinos afetados (Robinson et al., 2002). O clenbuterol tem efeito broncodilatador, aumentando o transporte mucociliar. Efeitos colaterais, tais como taquicardia e sudorese (Lavoie, 1997; Mair e Derksen, 2000), não foram observados nos três casos descritos. Nos casos com quantidade excessiva de muco nas pequenas vias aéreas, agentes mucocinéticos associados a broncodilatadores podem ser úteis para diminuir a resistência das vias aéreas e aumentar o transporte de mucociliar (Mair e Derksen, 2000). No presente relato, a associação do clenbuterol com a bromexina não apresentou efeito colateral.

O protocolo de tratamento nos três casos seguiu as recomendações da literatura, apresentando resultados satisfatórios. Os animais apresentaram melhora clínica a partir do sétimo dia de terapia, constatada pela diminuição dos episódios de tosse, secreção nasal, dispnéia expiratória, além da diminuição dos sibilos e das crepitações pulmonares.

Quando o eqüino susceptível é removido da pastagem ou do ambiente controlado para cocheira com poeira e é alimentado com feno, a obstrução das vias aéreas se desenvolve (Ainsworth e Biller, 2000; Mair e Derksen, 2000). Fato esse comprovado nos três casos após o desafio ambiental. Essa obstrução é resultante do broncoespasmo, acúmulo de muco e alterações inflamatórias na parede das vias aéreas (Robinson et al., 1993; Robinson, 1997).

Bronquiolite bacteriana secundária pode ocorrer em alguns casos de RAO, produzindo sinais clínicos de febre, descarga nasal purulenta, anorexia, neutrofilia e hiperfibrinogenemia (Mair e Derksen, 2000), entretanto tal complicação não foi observada nos três casos do presente relato.

Após o desafio ambiental, recomendou-se que os animais fossem mantidos soltos em piquetes com forragem adequada, com cocheiras de livre acesso durante quatro meses para promover a completa remissão dos sinais clínicos. Terminado esse período, os animais retornaram às cocheiras, sem apresentarem sintomatologia clínica de RAO

Os três muares continuaram a desempenhar suas atividades atléticas de modo satisfatório, embora com rendimento inferior ao observado antes do surgimento da enfermidade.

\section{REFERÊNCIAS BIBLIOGRÁFICAS}

AINSWORTH, D.M.; BILLER, D.S. Sistema respiratório. In: REED, S.M.; BAYLY, W. M. Medicina interna eqüina. Rio de Janeiro: Guanabara Koogan, 2000. p.217-249.

ART, T.; DUVIVIER, D.H.; VOTION, D. et al. Does an acute COPD crisis modify the cardiorespiratory and ventilatory adjustments to exercise in horses? J. Appl. Physiol., v.84, p.845$852,1998$.

DAVIS, E.; RUSH, B.R. Equine recurrent airway obstruction: pathogenesis, diagnosis, and pacient management. Vet. Clin. N. Am.: Equine Pract., v.18, p.453-467, 2002.

DERKSEN, F.J. Chronic obstructive pulmonary disease (heaves) as an inflammatory condition. Equine Vet. J., v.25, p.257-258, 1993.

HOLCOMBE, S.J.; JACKSON. C.; GERBER, $\mathrm{V}$. et al. Stabling is associated with airway inflammation in young Arabian horses. Equine Vet. J., v.33, p.244-9, 2001.

LAVOIE, J.P. Chronic obstructive pulmonary disease. In: ROBINSON, N. E. Current therapy in equine medicine. 4ed. Philadelphia: W. B. Saunders, 1997. p.431-435.

LÉGUILLETTE, R. Recurrent airway obstruction - heaves. Vet. Clin. N. Am.: Equine Pract., v.19, p.63-86, 2003.

MAIR, T.S.; DERKSEN, F.J. Chronic obstructive pulmonary disease: a review. Equine Vet. Educ., v.12, p.35-44, 2000. 
MOORE, B.R. Lower respiratory tract disease. Vet. Clin. N. Am.: Equine Pract., v.12, p.457472, 1996.

NYMAN, G.; LINDBERG, R.; WECKNER, D. et al. Pulmonary gas exchange correlated to clinical signs and lung pathology in horses with chronic bronchiolitis. Equine Vet. J., v.23, p.253$260,1991$.

ROBINSON, N.E.; DERKSEN, F.J.; BERNEY, C. et al. The airway response of horses with recurrent airway obstruction (heaves) to aerosol administration of ipratropium bromide. Equine Vet. J., v.25, p.299-303, 1993.

ROBINSON, N.E.; DERKSEN, F.J.; BUECHNER-MAXWELL, M.A. The pathogenesis of chronic obstructive pulmonary disease of horses. Br. Vet. J., v.152, p.283-306, 1996.

ROBINSON, N.E. Structure and function of the tracheobronquial system. In: ANNUAL MEETING OF AMERICAN ASSOCIATION EQUINE PRACTITIONERS, 43, 1997, Phoenix. Proceedings... Phoenix: AAEP, 1997. p.87-94

ROBINSON, N.E.; OLSZEWSKI, M.A.; BOEHLER, D. et al. Relationship between clinical signs and lung function in horses with recurrent airway obstruction (heaves) during a bronchodilator trial. Equine Vet. J., v.32, p.393400, 2000.
ROBINSON, N.E.; JACKSON, C.; JEFCOAT, A. et al. Efficacy of three corticosteroids for the tretment of heaves. Equine Vet. J., v.34, p.17-22, 2002.

ROBINSON, N.E.; BERNEY, C.; EBERHART, $\mathrm{S}$. et al. Coughing, mucus accumulation, airway obstruction, and airway inflammation in control horses and horses affected with recurrent airway obstruction. Am. J. Vet. Res., v.64, p.550-557, 2003.

RUSH, B. R. Immune theraphy in respiratory disease. Vet. Clin. N. Am.: Equine Pract., v.13, p.531-548, 1997.

THIEMANN, A.K.; BELL, N.J. The peculiarities of donkey respiratory disease. In: LEKEUX, P. Equine respiratory disease. International Veterinary Information Service, 2001. Disponível in: < http://www.ivis.org/special_books/Lekeux/bell>. Acessado em: 20 jun. 2006.

TREMBLAY, G.M.; FERLAND, C.; LAPOINTE, J-M. et al. Effect of stabling on bronchoalveolar cells obtained from normal and COPD horses. Equine Vet. J., v.25, p.194-197, 1993.

VANDENPUT, S.; DUVIVIER, D.; VOTION, D. et al. Environmental control to maintain stabled COPD horses in clinical remission: effects on pulmonary function. Equine Vet. J., v.30, p.93-96, 1998. 